\title{
Objects detection and tracking using fast principle component purist and kalman filter
}

\author{
Hadeel N. Abdullah, Nuha H. Abdulghafoor \\ Department of Electrical Engineering, University of Technology, Iraq
}

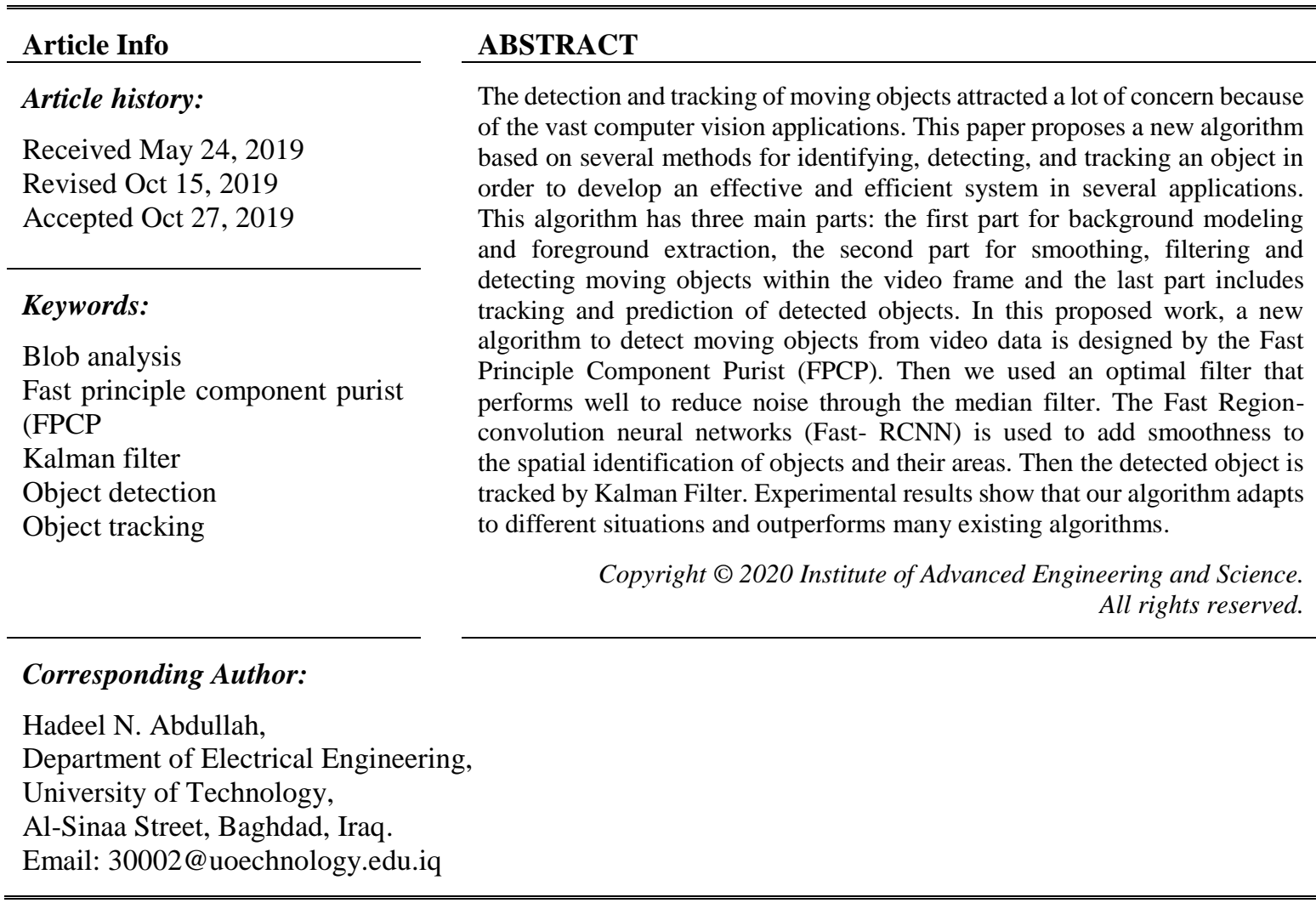

\section{INTRODUCTION}

The process of tracking is one of the main tasks in computer vision [1]. It has an important turn in many areas of research, such as movement objects, recognition and analysis human and nonhuman Vitality, 3D representation, mobility in vehicles, and others. Object tracking is the most common in automated monitoring applications because the individual human employer cannot manage the controlled area, especially when the number of cameras raises. Also, in the medicinal application, the operator cannot sometimes analyse the video taken by the device. It is also used in security systems, traffic management systems, and others. The tracking system can track single or multiple animation objects in different environments [2]. In general, the object detection and tracking system includes the different stages like background subtraction, object detection, and object tracking as shown in the block diagram in Figure 1.

In many fields of image processing and computer vision applications, the background modeling is an important step, also known as foreground detection, which extracts the foreground of the image for subsequent processing (such as object selection and identification) from background. These are the most important areas of the picture, which are called objects such as humans, cars, texts, etc. This stage may be after the preprocessing phase of the image, which may include noise reduction of images, and before the subsequent treatment phase such as morphology, etc [3]. A more common way to detect moving objects in videos is background subtraction. The basic principle is to detect moving objects from the difference between the current frame and the reference frame, often called a "background image" or "background model". Some common 
methods in this area include the use of frame differentiation, optical flow, analysis of principal components, background mixture models [4].

The foreground represents the object of interest in a scene. To represent an object, features are extracted that uniquely defines an object. These features or the descriptors of an object are then used to track the object. A feature is an image pattern that differentiates an object from its neighborhood. The features of an object are converted into descriptors, also referred to as appearance features, using some operations around the features [2]. The commonly used object representations for tracking are centroid, multiple points, rectangular patch, and complete object contour, etc. while the descriptors of an object such as probability densities of object appearance (Histogram), template, blob analysis, etc.

The object tracking is to select and give individual paths to each object in the video sequence. Objects can be humans on the street, cars on the road, players on the pitch, or from a group of animals. The object is tracked to extract the object, identify the object and track it, and the decisions related to their activities. Trace objects can be classified as points tracking, kernel tracking, and trace shadow images. The general techniques of tracking such as Kalman Filter, Particle Filter, Mean Shift Method, etc [5].

The main contribution of this work is:

a. The proposed algorithm uses the FPCP technique to extract the motion areas from different backgrounds of the captured video frame without the need for further input. As a result, so the outputs have good speed and accuracy.

b. Using the method of analysing the spatial and temporal simultaneously to select the effective pixels in the motion zones and to determine the area of the object at the same time using Fast RCNN. In addition to using efficient tracking technique, Kalman Filter is thus an efficient and integrated way to track multiple objects in the same captured video frame.

This paper is ordered as follows. Section 2 explains the related works. Section 3 explains Methodologies (Mathematical Background), Section 4 explains the proposed Algorithm. Section 5 explains the results and performance analysis. Finally the conclusions in Section 6.

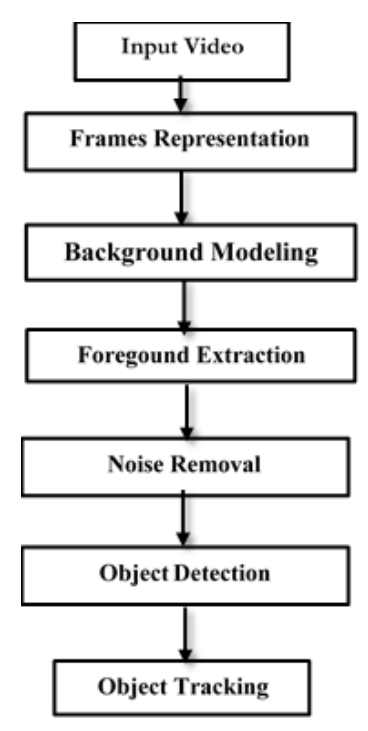

Figure 1. The block diagram of object and tracking system

\section{RELATED WORKS}

Since the last few decades, many researchers have proven algorithms for detecting and tracking objects. In this section, we demonstrated some of these algorithms related to the proposed system. According to [6], motion brim is extracted in polar-log coordinate, then the gradient operator is employed to compute the optical flow directly in every motion regions, then the object is tracked. In the proposed work in [7], the active background reconstructed and the object size determined as a preliminary task, to extract and track the object in the foreground .The method in [8] is the object detection is done by Gaussian Mixture Model (GMM), and the tracking is done by Kalman Filter. In this method, Object detection is determined based on the size of the foreground. Therefore Errors will occur in determining the object such as the object and its shadow are merged as an object or representing two adjacent compounds as a single object. The paper [9] 
developed; the algorithm includes optical flow and the motion vector estimation for object detection and tracking. The detection and tracking system in [10] is sophisticated depend on optical flow for detection; the object tracking is done by blob analysis.

In Prabhakar et al. [11], a moving object tracking system using morphological processing and blob analysis, which able to distinguish between car and pedestrian in the same video. In the paper [12], the foreground is extracted from the background using multiple-view architecture. After that, the forward movement date and editing schemes are used to detect the animated objects. Finally, by detecting the center of gravity of the moving object, it is used to trace the object based on the Kalman filter. In the method [13], animated objects are represented as groups of spatial and temporal points using the Gabor 3D filter, which works on the spatial and temporal analysis of the sequential video and is then joint by using the Minimum Spanning Tree. The proposed technique described in [14], split into three stages; Foreground segmentation stage by using Mixture of Adaptive Gaussian model, tracking stage by using the blob detection and evaluation stage which includes the classification according to the feature extraction. The proposed work in [15], it merged Background Subtraction with Low Rank techniques for effective object detection. Finally the suggested algorithm in [16], it employed Background Subtraction and K-Means Clustering techniques to detect the moving object and tracking it. After exploring some of the published research on the detection and tracking of the object, it was found that the discovery and tracking of the object is a complex task because of many elements of dynamic tracking such as determining the type of camera moving or static, the random change of the speed of the object, the intensity of light and darkness, etc.

\section{METHODOLOGIES (MATHEMATICAL BACKGROUND)}

\subsection{Fast Principal Component Pursuit}

FPCP was recently suggested as a powerful alternative to Principal Components Analysis (PCA). This method will be used in various applications, including foreground/background modelling, data analysis, whether in text or video format and image processing. The PCA was formulated initially [17].

$$
\arg \min _{L, S}|| L\left\|_{o}+\lambda\right\| S \|_{1} \quad \text { s.t. } \quad D=L+S
$$

Where $D \in R^{m \times n}$ is the observed matrix, $\|L\|_{o}$ is the nuclear norm of matrix $L$ (i.e. $\sum_{k}\left|\sigma_{k}(L)\right|$ ) and $\|\mathrm{S}\|_{1}$ is the $1^{1}$ norm of matrix $S$. Numerous changes have been made to eq. (1) by changing the restrictions on sanctions and vice versa. So that the eq. (1) became:

$$
\underset{t}{\arg \min _{L, S}} \frac{1}{2}|| L+S-D\left\|_{F}+\lambda\right\| S \|_{1} \text { s.t. }\|L\|_{o}<
$$

The constraint $\|\mathrm{L}\|_{\mathrm{o}}<\mathrm{t}$ is active, represents a constraint of equality, so it is suggested that the algorithm ranks the same rather than relax the nuclear base, so the function is as follows

$$
\arg \min _{L, S} \frac{1}{2}|| L+S-D\left\|_{F}+\lambda\right\| S \|_{1} \quad \text { s.t. } \quad \operatorname{rank}(L) \approx t
$$

This adjustment ignored the initial selection of the parameter $\lambda$, the background modelling compound $L$ is often low, and in practice, there is no difficulty in selecting the appropriate value for $t$. A normal process to solve (3) is the alternative minimization:

$$
\begin{aligned}
& L_{k+1}=\arg _{L} \min \left\|L+S_{k}-D\right\|_{F} \quad \text { s.t. } \operatorname{rank}(L) \approx t \\
& S_{k+1}=\arg _{S} \min \left\|L_{k+1}+S-D\right\|_{F}+\lambda\|S\|_{1}
\end{aligned}
$$

The (3) can be solved by taking a partial Singular Value Decomposition SVD of (D - $S_{k}$ ) with respect to t. while the eq. 4 can be solved by element-wise shrinkage. The background of the videos is supposed to lie in a low-level sub-space, and the moving objects should be in the foreground as if they were gradually soft in the spatial and temporal direction [18]. The proposed method integrates the Frobenius and $1^{1}$-norm base into a unified framework for simultaneous noise reduction and detection. The Frobenius base uses the low-level property in the background; the contrast is improved by the $1^{1}$ norm standard. 


\subsection{Noise filtering}

Animated digital pictures often overlap with a set of noise based on prevailing conditions. Some of this noise is very disturbing when implicated in altering the intensity of video frames. It spoils pixels randomly and divides into two extreme levels: relatively low or relatively high, compared to adjacent pixels [11]. Subsequently, it is necessary to apply refinement technicalities that are able to handle various types of noise. Morphological processes are performed to extract important features of useful images in the impersonation of shapes in the region and their description. We have used both the morphology of the closure and corrosion, respectively, to remove parts of the road and unwanted things. After the morphological closure process, provided that the appearance of the object is not destroyed, and that many small punctures and separate pixels are filled in the form of one large real object. The following is the definition of morphological closure process and the applicable structural element B.

$$
\mathrm{P} * \mathrm{~B}=(\mathrm{P} \oplus \mathrm{B}) \oplus \mathrm{B}
$$

Where:

$$
B=\left[\begin{array}{lll}
0 & 0 & 1 \\
0 & 1 & 0 \\
1 & 0 & 0
\end{array}\right]
$$

The matrix $\mathrm{P}$, which includes moving object information, is obtained through the detection process. An integral part of the morphological expansion and erosion processes is a structural element of a flat shape. There is a binary flat structure element with a living value, either 2-D or multi-dimensional, in which the real pixels are included in the morphological calculation, and false pixels are not. The middle pixel of the structure element, called the parent, determines the pixel in the image being processed [11]

\subsection{Fast-region convolution neural network}

The reference to the recent advantages of convolutional neural networks (CNN) has been very successfulin a variety of computer vision tasks, especially those associated with detecting objects. In this research, we use CNN networks to identify the commercial object as a supervised learning task [19]. The Fast R-CNN Object Detection tool (Regions with Convolutional Neural Networks) is used to locate detected objects that are returned as a set of peripheral boxes. The detector has high confidence in the discoveries. Annotate the image with the surrounding squares of the detector and the corresponding detection grades. These peripheral boxes were called area suggestions or object suggestions. Zone suggestions were just lists of square boxes with a small probability of containing an object. The frame work of Fast R-CNN as shown in Figure 2 [20]. In many applications where the calculation is time-consuming, one can use point analysis to eliminate points that do not matter based on specific spatial properties and retain relevant points for further analysis The object corresponding to the point area is detected as a composite object and features as a bounded box.

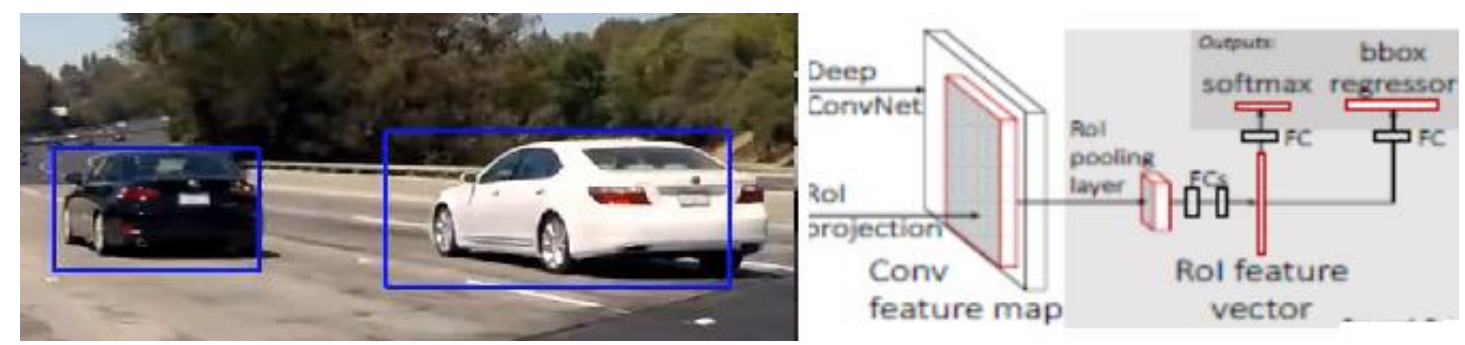

Figure 2. The frame work of Fast R-CNN

\subsection{Kalman filter}

Object tracking is a way to find and create a path to the object that was discovered. In this search, the Kalman filter method was used to track an object in sequence with captured video [21]. The Kalman filter is a linear approach that operates in two basic phases of prediction and correction (update). The prediction phase is accountable for the scoop of the next state and position of the present object. However the correction phase provides the parameters with their instance, they combine the actual measurement with the previous 
estimate to improve the trajectory where the object information detected in the previous frame is used and provides an estimate of the object's new position. The Kalman filter has the ability to rating the tracking locations with minimal datum on the location of the object. Initially, the status St and measurement Xt paradigm are determined to predict the next site as shown in Figure 3.

The Kalman filter is a repetitive algorithm to estimate the evolving state of a process when making measurements in the process. The Kalman filter is linear when the evolution of the case follows the linear motion model and the measurements are linear functions of the case. The Kalman filter can accurately track motion based on adaptive filtering using the state area model. It is necessary to design a dynamic model of the target movement which is the fixed speed model $(\mathrm{CV})$, which assumes that the speed is constant during the sampling period. This model has been used in various applications for its ease and effectiveness [22].

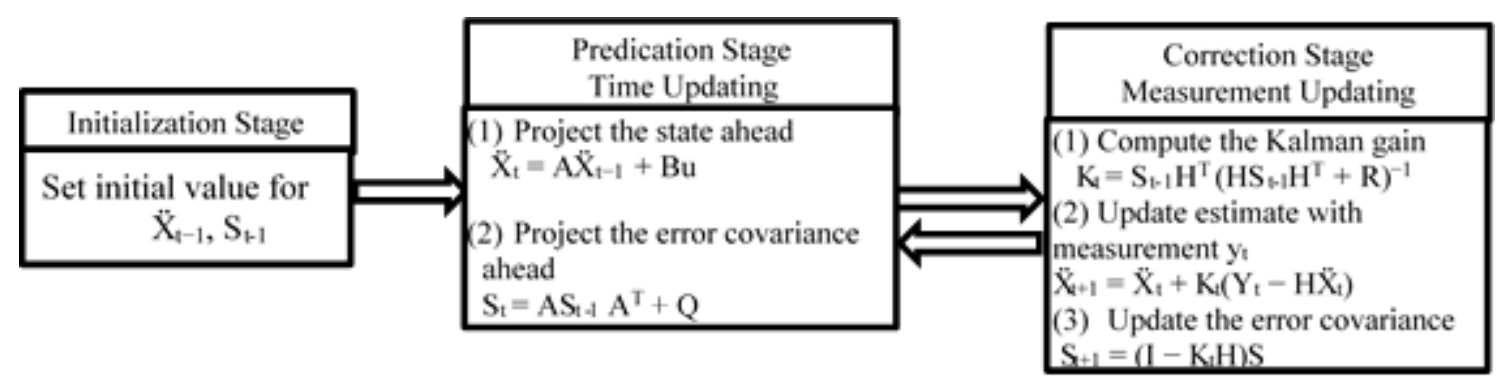

Figure 3. The block diagram of Kalman Filter Algorithm

Where; A - state transition matrix, B - coverts control input, Q - process noise covariance K- Kalman gain, $\mathrm{X}$ - measurement matrix, $S$ - measurement error covariance and $\mathrm{H}$ - model matrices. The prediction of the next state $\mathrm{S}_{\mathrm{t}+1}$ is done by integrating the actual measurement with the pre-estimate of the situation $\mathrm{S}_{\mathrm{t}-1}$.

\section{THE PROPOSED SYSTEM}

In this pager, object detection and tracking algorithm, a collection of two famous computer visibility technologies, Fast Principle Component Purist (FPCP) and the Kalman filter, was introduced. FPCP is used in the object discovery phase. It provides quick and delicate object detection on other methods such as background subtraction. FPCP does not provide the path of motion, instead it supplies acquaintance about the orientation of the object and its motion in vector form. The Proposed algorithm of FPCP as shown in Algoritm1.

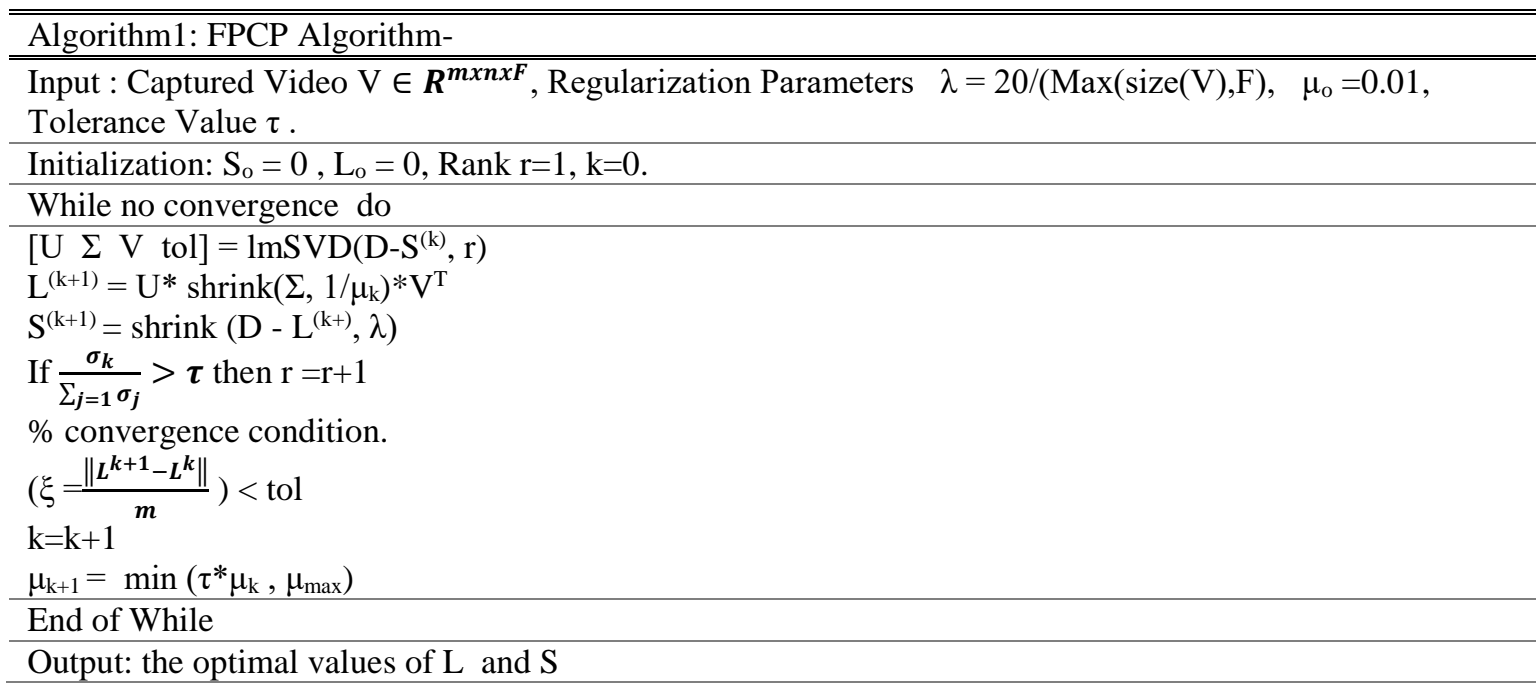

This algorithm has a simpler and clearer style. Since the video background modeling application component L is usually very low, we suggest a simple procedure for estimating the upper limit of r, so we can estimate the contribution of the new single vector; if this input is small enough, we stop increasing the value of $\mathrm{r}$. In our experimental simulations, this corresponds to an estimate of the order made by the inexact ALM. This algorithm is used LMSVD (Limited Memory Singular Value Decompositions). This function is based on 
a block Krylov subspace optimization technique which significantly accelerates the classic simultaneous iteration method [23]. Our computational results also show that our proposed algorithm has low memory space that can be applied in real-time video applications, faster than modern applications and deliver comparable quality results.

The proposed system as showin in Figure 4. It shows the detail stages of the system are followed to that deals with background separation, object and feature extraction. At first, the video is taken by the stationary camera. The video is only a series of cascading frames, so the object detection manner must first detect the moving object in these cascading frames. Then the algorithm converts the video into two dimension matrices to facilitate handling in mathematical calculations, to reduce the time calculation and memory requirementsThe processing includes removing the noise. Then the process status of all pixel is tested by Fast RCNN and clustered it to detect the object. Initialize the tracking stage and update the tracker in every new frame. This system has many features, including the possibility of tracking more than one object and the speed of response to a change in speed and change in the scale. Figure 4 shows the original video was converted to individual frames to be processed in next stages. The foregrouial nd was extrac ted by the FPCP detection and showed holes and noise on the frame. In order to clarify and soften the frame, the morphology was done. The foreground frame is shown after the morphological process and the final output frame which includes tracked objects with bounding boxes.
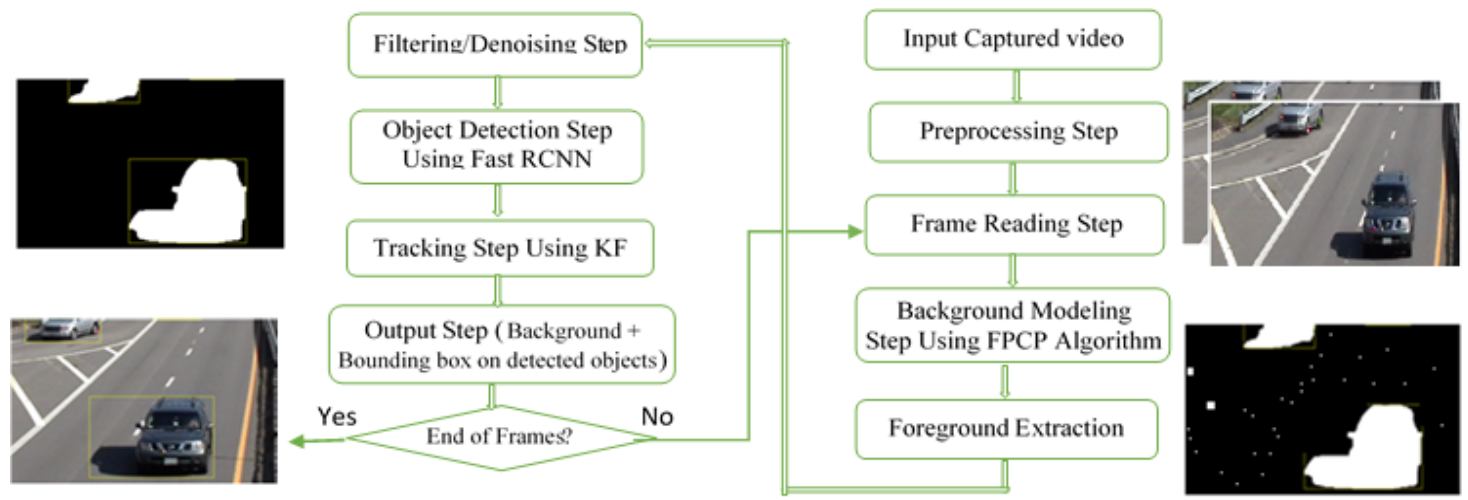

Figure 4. The fow chart of the proposed algorithm

\section{RESULTS AND PERFORMANCE ANALYSIS}

The algorithm proposed in MATLAB (2018b) has been applied, and their experiments were performed on a Computer type MSI GV63 with Intel Core i7 8750H, NVIDIA RTX 2060 6G, 256GB SSD+1TB and 16 GB RAM. It has three stages are foreground detection, filtering and tracking. The proposed algorithm detects the movable objects accurately and keeps track of their appearance in the sequence video frames. Video data has been used in any format as an input to the proposed work, and good results have been obtained in various article conditions on this indoor, outdoor, light traffic and dense traffic. The efficiency of the proposed algorithm was evaluated, the experiential outcomes were as follows

Several sampled video is used in the various environment in order to test the performance of the proposed algorithm. The experiential outcomes are given in Figure 5. The first column as shown in Figure 5(a) shows the sampled frames of the video then the second column as shown in Figure 5(b) shows a clean foreground extracted frames by FPCP detection are given. The third column as shown in Figure 5c) includes the detected and tracked objects by marking it with a circumferential box. Table 1 explains the mean execution times $(\mathrm{sec})$ of the proposed system when implemented on 365 sampled video frames by Matlab version 2018b. Accuracy is a measure of the performance efficiency of the object tracking system. The detection and tracking system precision can be calculated using the next formula:

$$
\text { Accuracy }=\frac{\text { The total number of detected objects by system }}{\text { The total number of actual objects in video }}
$$



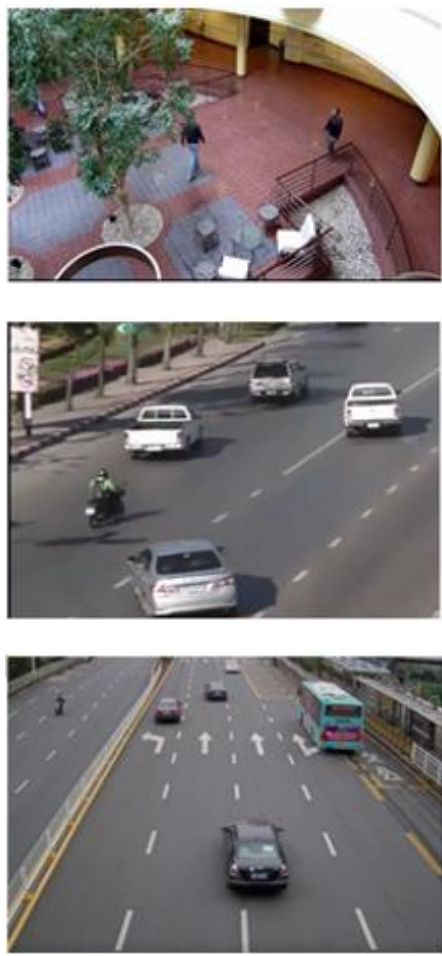

(a)
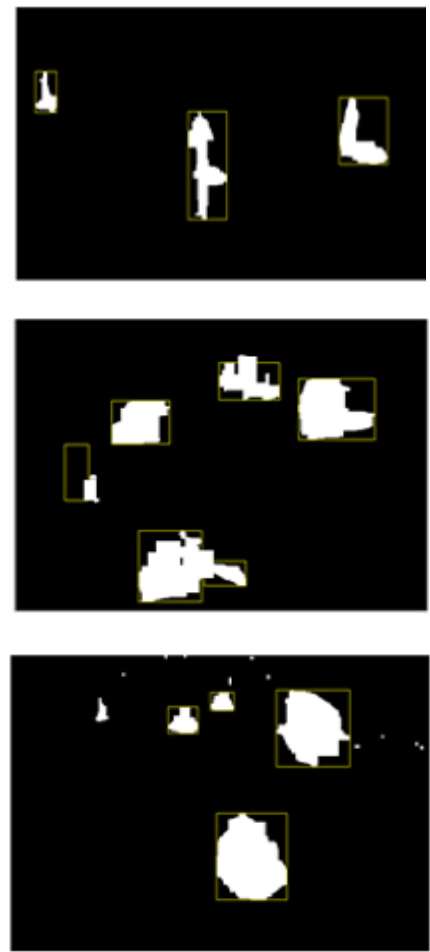

(b)
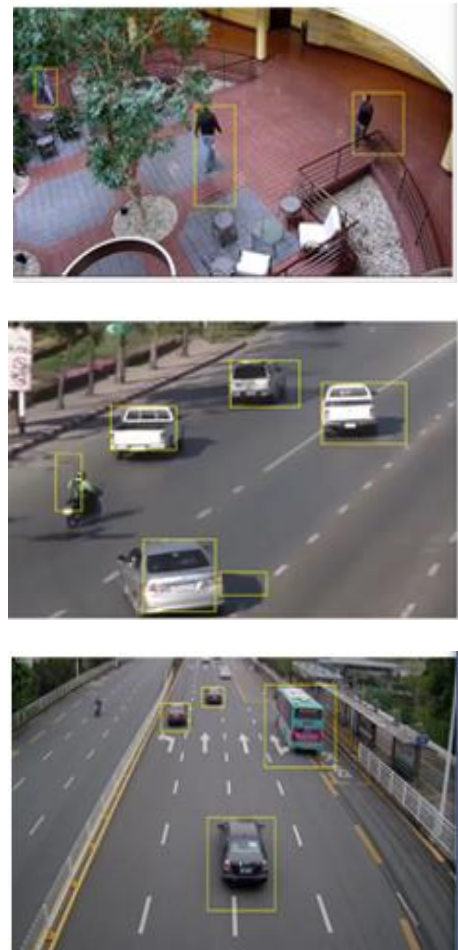

(c)

Figure 5. The proposed algorithm results on several captured video,

(a) Original framq, (b) The filtered foreground and (c) The resultant frame

The proposed algorithm for the different video input has been tested with different methods to evaluate its accuracy. The accuracy of the proposed tracker in different input scenes was compared and compared with other tracking systems, as shown in Table 2. It shows that the detection and tracking accuracy rate using the proposed algorithms is $100 \%$. The results are optically acceptable except for an algorithm that proves that this multi-object tracking method is validated. It was concluded that the proposed algorithm was still competitive, although some results were closest to another, with little degradation in some as a result of the cost of some complex calculations. The detection precision of the suggested algorithm is compared with other known and present methods. The comparison shows the efficient performance of the proposed method on some of the selected frames shown in Figure 6. We compared the proposed algorithm with the most representative algorithms and for different frame sizes and settings for the tested videos; we used gray or chromatic video sequences. The results were comparable to the proposed algorithm with other algorithms.

Table 1. Performance execution time

\begin{tabular}{lc}
\hline The Step & Mean Execution Time (Second) \\
\hline Loading the video and conversion to matrix & 9 \\
Preprocessing and frame reading & 5.2 \\
Foreground detection & 12.2 \\
Noise Removal & 1.15 \\
The detection process & 6.2 \\
Tracking System & 4 \\
Total & 37.75 \\
\hline
\end{tabular}

Table 2. Percentage accuracy \%

\begin{tabular}{lcccc}
\hline Comparison Parameter & $\begin{array}{c}\text { Bachground } \\
\text { Subtraction [15] }\end{array}$ & $\begin{array}{c}\text { GMM Method } \\
{[8]}\end{array}$ & $\begin{array}{c}\text { Optical Flow } \\
\text { Method [10] }\end{array}$ & $\begin{array}{c}\text { Proposed Tracking } \\
\text { System }\end{array}$ \\
\hline Single Human & 100 & 100 & 90 & 100 \\
Speed Diversity & 80 & 80 & 10 & 85 \\
View Point Difference & 85 & 90 & 90 & 90 \\
Fixed Objects & 75 & 80 & 70 & 90 \\
Multiple Objects & 90 & 85 & 20 & 95 \\
\hline
\end{tabular}


To evaluate the visual performance of the proposed algorithm, we compared the proposed algorithm to 3 algorithms. The videos examined contain different background scenes, and multiple moving objects both outdoors and indoors (pedestrians, vehicles, etc.). We have chosen the following most methods to compare with our proposed method: (1) The Background Subtraction (BG SUB) [15], (2) GMM method [8], and (3) Optical Flow (OF) [10]. Visual results are shown on the videos tested in Figure 6. Individual and group infantry, small dynamic background, and multiple traffic surveys, as shown in Figure 6, the proposed algorithm is closest to Ground Truth (GT). Some of the results of the tested algorithms consider the foreground object as the background. The main reason is that the parts of the object remain static in the video, and that the proposed algorithm has overcome this effect, and obviously the detection effect is better than other algorithms.

In order to enhance the robustness and efficiency of the proposed system, a second comparison was made between Kalman Filter Tracker and Mean Shift Tracker [24] as shown in Figure 7. The Mean Shift algorithm lists the non-parametric density that finds that the picture frame is very similar to the color histogram of the object in the current frame. Mean-Shift tracking frequently increases appearance similarity by comparing the object layout and the window layout around the location of the filter object. This algorithm has the ability to track in real time, the nonlinear moving objects, and has good applicability with object distortion and rotation. However, the Mean Shift algorithm does not use the object's direction and speed information to track an object, and it is easy to lose an object when there is interference (such as light and scattering) in the surrounding environment and cannot deal with scale and clutter differences [25]. To achieve a good trace effect, it is extremely important to improve the performance of the trace algorithm by choosing a distinct feature to create the object model, so that the characteristics of the object and the background are clearly different. Table 3 shows some advantages and disadvantages of both methods.
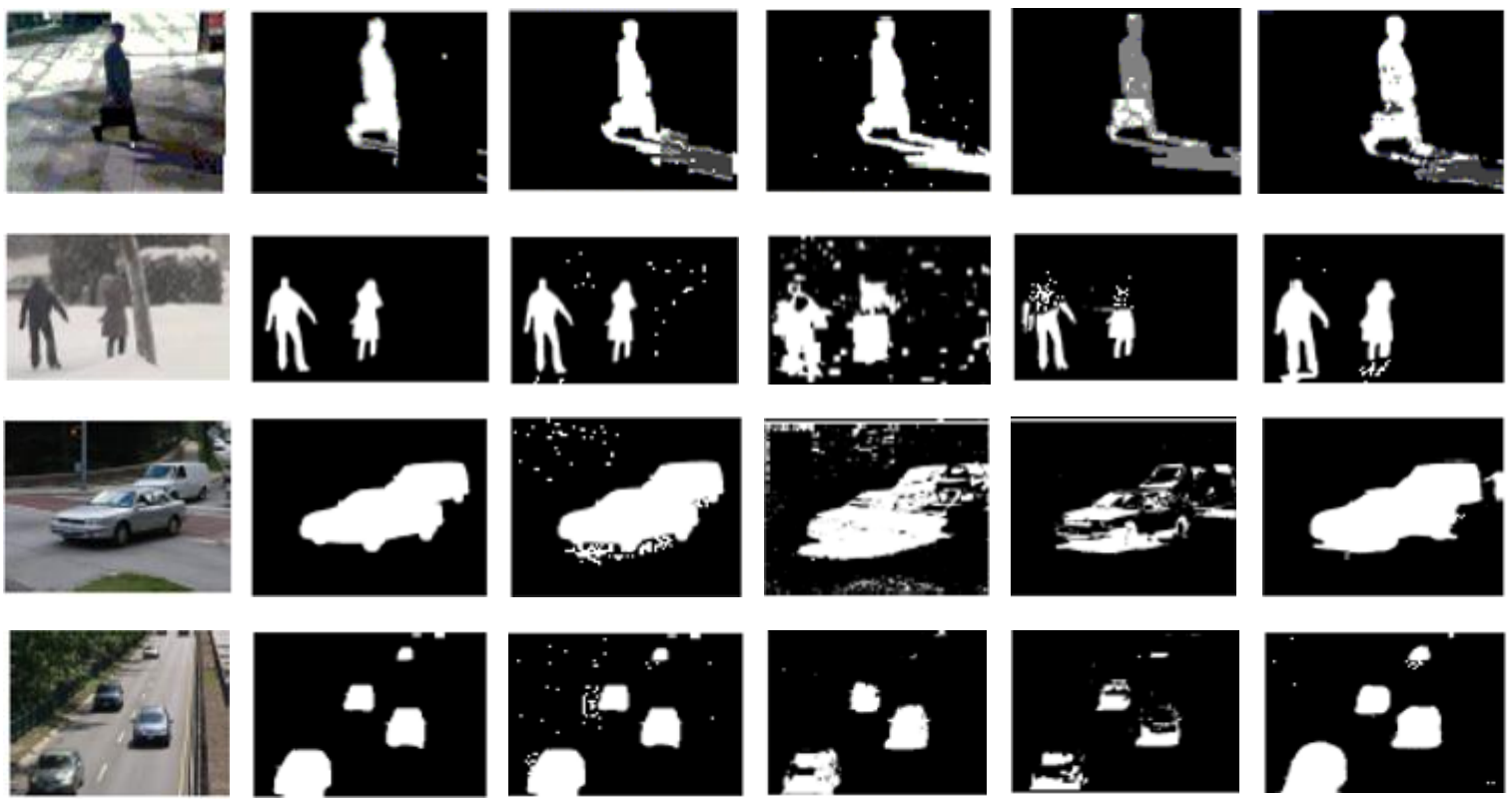

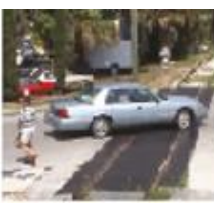

(a)

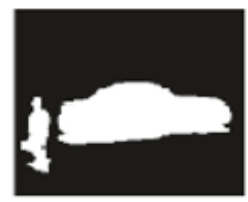

(b)

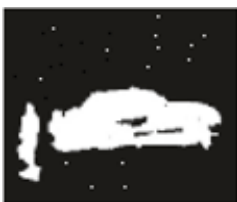

(c)

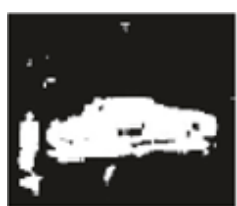

(d)

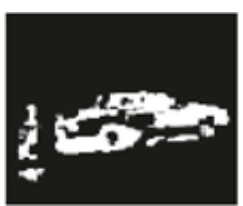

(e)

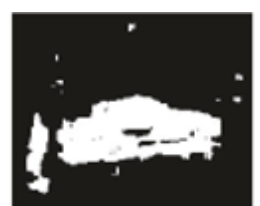

(f)

Figure 6. The comparison results for several experiment captured video: (a) Input Frame (I/P), (b) The Ground Truth (GT), (c) The background Subtraction (BG SUB ) [15] (d) GMM Method [8], (e) Optical Flow (OF) [10], and (f) The Proposed Algorithm (FPCP) 


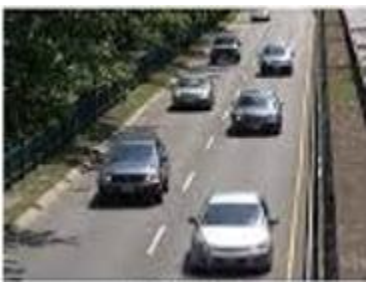

(a)

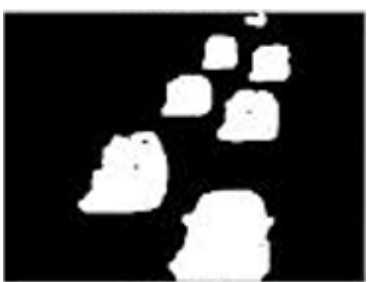

(b)

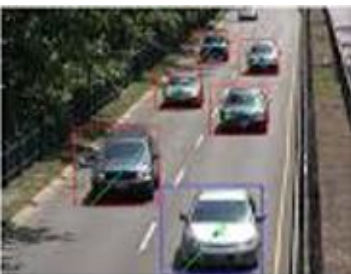

(c)

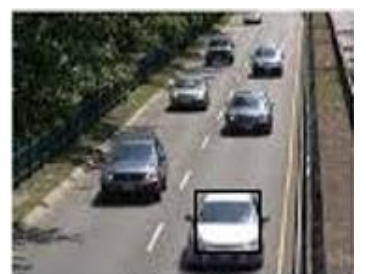

(d)

Figure 7. The comparison results for several tracking techniques:

(a) Original Frame, (b) object Detection, (c) KF Tracking, and (d) Mean Shift Tracking [24]

Table 3: Comparison between Kalman Filter and Mean Shift Tracker

\begin{tabular}{cll}
\hline Technique & Advantages & Disadvantages \\
\hline Kalman Filter & $\begin{array}{l}\text { 1. Easy to implement, Point tracking } \\
\text { (Distance and Velocity). }\end{array}$ & $\begin{array}{l}\text { 1. The computationally expensive. } \\
\text { 2. Continuous tracking despite pauses in moving } \\
\text { videos. }\end{array}$ \\
& $\begin{array}{l}\text { 3-It can track multiple objects. } \\
\text { 1. It tracks based on color of object } \\
\text { 2. It is nonlinear tracker }\end{array}$ & $\begin{array}{l}\text { 1. Ineffective when there are occlusion or distortion } \\
\text { problems. }\end{array}$ \\
& 2 A single object tracker. \\
\hline
\end{tabular}

\section{CONCLUSION}

The algorithm provided was implemented in Matlab. Frames are processed in different sizes using msi type Intel Core i7 $8750 \mathrm{H}$ computer. The proposed algorithm shows its advantage over existing object methods more precisely from the results obtained.The proposed algorithm was compared with existing video file algorithms where the results show the efficiency of the proposed work. The proposed system can process gray and color videos at few seconds per GPU-Matlab application frame. The proposed algorithm can adapt to background changes. Object detection and tracking are main and affront mission in many computer visibility implementations, such as monitoring, car saltworks, routing, and automation. This algorithm presents several benefits, such as multiple object detection and tracking in different environments. The disadvantages of this technique using one method will not produce perfect results because its accuracy is influenced by different operators such as the low resolution of captured video, change in weather. Etc. In the future, we hope to expand our scope of detection and tracking of objects in overcrowded scenery or the appearance of severe contrast in lighting and real-time scenes.

\section{REFERENCES}

[1] Cheng, J., Yang, J., Zhou, Y. and Cui, Y., “Flexible background mixture models for foreground segmentation," Image and Vision Computing, 24(5): 473-482, 2006.

[2] Wu, Y., Lim, J. and Yang, M.H., "Online object tracking: A benchmark," In Proceedings of the IEEE conference on computer vision and pattern recognition (pp. 2411-2418). 2013.

[3] Naeem, H., Ahmad, J. and Tayyab, M., "Real-time object detection and tracking," In INMIC (pp. 148-153). IEEE, December 2013.

[4] Cyganek, B., "Object detection and recognition in digital images: theory and practice, ” John Wiley \& Sons, 2013.

[5] Tang, S.L., Kadim, Z., Liang, K.M. and Lim, M.K., "Hybrid blob and particle filter tracking approach for robust object tracking," Procedia Computer Science, 1(1), 2549-2557, 2010.

[6] Zhang, H.Y., "Multiple moving objects detection and tracking based on optical flow in polar-log images," In International Conference on Machine Learning and Cybernetics (vol. 3, pp. 1577-1582), July 2010.

[7] Mandellos, N.A., Keramitsoglou, I. and Kiranoudis, C.T., "A background subtraction algorithm for detecting and tracking vehicles," Expert Systems with Applications, 38(3): 1619-1631, 2011.

[8] Bakti, R.Y., Areni, I.S. and Prayogi, A.A., "Vehicle Detection and Tracking using Gaussian Mixture Model and Kalman Filter," International Conference on Computational Intelligence and Cybernetics (pp. 115-119), IEEE, November 2016.

[9] Kale, K., Pawar, S. and Dhulekar, P., "Moving object tracking using optical flow and motion vector estimation," In 4th International Conference on Reliability, Infocom Technologies and Optimization (ICRITO)(Trends and Future Directions) (pp. 1-6), IEEE, September 2015.

[10] Aslani, S. and Mahdavi-Nasab, H., "Optical flow based moving object detection and tracking for traffic surveillance." International Journal of Electrical, Electronics, Communication, Energy Science and Engineering, 7(9): 789-793, 2013. 
[11] Telagarapu, P., Rao, M.N. and Suresh, G., "A novel traffic-tracking system using morphological and Blob analysis," In International Conference on Computing, Communication and Applications (pp. 1-4). IEEE, February 2012.

[12] Hu, W.C., Chen, C.H., Chen, T.Y., Huang, D.Y. and Wu, Z.C., "Moving object detection and tracking from video captured by moving camera," Journal of Visual Communication and Image Representation, 30: 164-180, 2015.

[13] Ray, K.S. and Chakraborty, S., "Object detection by spatio-temporal analysis and tracking of the detected objects in a video with variable background," Journal of Visual Communication and Image Representation, 58: 662-674. 2019.

[14] Mahalingam, T. and Subramoniam, M., "A robust single and multiple moving object detection, tracking and classification," Applied Computing and Informatics, 2018.

[15] Cao, W., Wang, Y., Sun, J., Meng, D., Yang, C., Cichocki, A. and Xu, Z., "Total variation regularized tensor RPCA for background subtraction from compressive measurements," IEEE Transactions on Image Processing, 25(9): 40754090, 2016.

[16] Supreeth, H. S. G., and Chandrashekar M. Patil. "Efficient multiple moving object detection and tracking using combined background subtraction and clustering," Signal, Image and Video Processing, 12(6): 1097-1105, 2018.

[17] Bouwmans, T. and Zahzah, E.H., "Robust PCA via principal component pursuit: A review for a comparative evaluation in video surveillance," Computer Vision and Image Understanding, 122: 22-34, 2014.

[18] Rodriguez, P. and Wohlberg, B., "Fast principal component pursuit via alternating minimization," In IEEE International Conference on Image Processingc (pp. 69-73), IEEE, September 2013.

[19] Zhao, Z.Q., Zheng, P., Xu, S.T. and Wu, X., “Object detection with deep learning: A review," IEEE transactions on neural networks and learning systems, 2019.

[20] Girshick, R., "Fast r-cnn." In Proceedings of the IEEE international conference on computer vision (pp. 1440-1448). 2015.

[21] Li, Q., Li, R., Ji, K. and Dai, W., "Kalman filter and its application," In International Conference on Intelligent Networks and Intelligent Systems (ICINIS) (pp. 74-77), IEEE, November 2015.

[22] Saho, K., "Kalman Filter for Moving Object Tracking: Performance Analysis and Filter Design," In Kalman FiltersTheory for Advanced Applications. IntechOpen, 2017.

[23] Liu, X., Wen, Z. and Zhang, Y., "Limited memory block Krylov subspace optimization for computing dominant singular value decompositions," SIAM Journal on Scientific Computing, 35(3): A1641-A1668, 2013.

[24] Shivhare, A. and Choudhary, V., "Object tracking in video using mean shift algorithm: A review," International Journal of Computer Science and Information Technologies, 2015.

[25] Karasulu, B. and Korukoglu, S., "Performance Evaluation Software: Moving Object Detection and Tracking in Videos," Springer Science \& Business Media, 2013. 